\title{
RAPID IMBIBITION OF WATER IN FRACTURES WITHIN UNSATURATED SEDIMENTARY ROCK
}

\author{
C.-L. Cheng ${ }^{1,2^{*}}$, E. Perfect ${ }^{3 *}$, B. Donnelly ${ }^{3}$, H.Z. Bilheux ${ }^{4}$, A.S. \\ Tremsin $^{5}$, L.D. McKay ${ }^{3}$, V.H. DiStefano ${ }^{6,7}$, J.C. Cai ${ }^{8}$, \\ and L.J. Santodonato ${ }^{9}$
}

${ }^{1}$ Department of Physics and Geology, University of Texas - Pan American, Edinburg, TX 78539, USA

${ }^{2}$ Department of Mechanical Engineering, University of Texas - Pan American, Edinburg, TX 78539, USA

${ }^{3}$ Department of Earth and Planetary Sciences, University of Tennessee Knoxville, Knoxville, TN 37996, USA

${ }^{4}$ Chemical and Engineering Materials Division, Oak Ridge National Laboratory, Oak Ridge, TN 37831, USA

${ }^{5}$ Experimental Astrophysics Group, Space Sciences Laboratory, University of California, Berkeley CA 94720, USA

${ }^{6}$ Bredesen Center for Interdisciplinary Research and Graduate Education, University of Tennessee - Knoxville, TN 37996, USA

${ }^{7}$ Chemical Sciences Division, Oak Ridge National Laboratory, Oak Ridge, TN 37831, USA

${ }^{8}$ Institute of Geophysics and Geomatics, China University of Geosciences, Wuhan 430074, P.R. China

${ }^{9}$ Instrument and Source Design Division, Oak Ridge National Laboratory, Oak Ridge, TN 37831, USA

* Corresponding author

e-mail: chengc@utpa.edu, phone: +1-956-665-2464 
The spontaneous imbibition of water and other liquids into gas-filled fractures in variably-saturated porous media is important in a variety of engineering and geological contexts. However, surprisingly few studies have investigated this phenomenon. We present a theoretical framework for predicting the 1-dimensional movement of water into air-filled fractures within a porous medium based on early-time capillary dynamics and spreading over the rough surfaces of fracture faces. The theory permits estimation of sorptivity values for the matrix and fracture zone, as well as a dispersion parameter which quantifies the extent of spreading of the wetting front. Quantitative data on spontaneous imbibition of water in unsaturated Berea sandstone cores were acquired to evaluate the proposed model. The cores with different permeability classes ranging from 50 to $500 \mathrm{mD}$ and were fractured using the Brazilian method. Spontaneous imbibition in the fractured cores was measured by dynamic neutron radiography at the Neutron Imaging Prototype Facility (beam line CG-1D, HFIR), Oak Ridge National Laboratory. Water uptake into both the matrix and the fracture zone exhibited square-root-of-time behavior. The matrix sorptivities ranged from 2.9 to $4.6 \mathrm{~mm} \mathrm{~s}^{-0.5}$, and increased linearly as the permeability class increased. The sorptivities of the fracture zones ranged from 17.9 to 27.1 $\mathrm{mm} \mathrm{s}^{-0.5}$, and increased linearly with increasing fracture aperture width. The dispersion coefficients ranged from 23.7 to $66.7 \mathrm{~mm}^{2} \mathrm{~s}^{-1}$ and increased linearly with increasing fracture aperture width and damage zone width. Both theory and observations indicate that fractures can significantly increase spontaneous imbibition in unsaturated sedimentary rock by capillary action and surface spreading on rough fracture faces. Fractures also inrease the dispersion of the wetting front. Further research is needed 
1 to investigate this phenomenon in other natural and engineered porous

2 media.

3

4 KEYWORDS

5

6 Neutron radiography; fractured porous media; unsaturated flow; capillarity;

7 fluid spreading; rough surfaces 


\section{INTRODUCTION}

The movement of water and other liquids in fractures within unsaturated earth materials is important in a variety of contexts, including the storage of hazardous wastes within the deep vadose zone, enhanced oil recovery, shale gas extraction, and water damage to the foundations of buildings and other structures. Advances in neutron, nuclear magnetic resonance, and $x$-ray imaging technologies have significantly improved our experimental capabilities to visualize fracture flow in variably-saturated porous media. As a result, researchers are now able to routinely quantify the distribution and movement of water within a wide range of natural and engineered materials at the spatial and temporal resolutions relevant to fracture flow.

While a large body of literature exists documenting water entry into gasfilled fractures under positive water pressures (e.g., Chen and Kinzelbach, 2002; Rangel-German and Kovscek, 2002; Karpyn et al., 2009; Kanematsu et al., 2009; Polsky et al., 2013), surprisingly few studies have investigated water uptake into fractures when the gauge pressure of the water is zero or less. These studies have involved either fractured cementitious materials (concrete, engineered cementitious composites, and mortar) or rocks (granodiorite and sandstone).

The rapid penetration of water into individual air-filled fractures in steelreinforced concrete was visualized using neutron imaging by Zhang et al. (2010a). Şahmaran and Li (2009) found through sorptivity testing that micro-cracks induced by mechanical loading increased the rate of water uptake in engineered cementitious composites. Zhang et al. (2010b; 2011) confirmed this finding using neutron radiography, while Snoeck et al. (2012) and Van Tittelboom et al. (2013) employed the same method to investigate the crack sealing efficiency of various water repellant chemicals on capillary uptake in fractured mortar mixtures. 
1 An early neutron imaging study of water imbibition into a granodiorite 2 rock core containing a fault-gouge filled fracture was conducted by Lunati et 3 al. (2003). Due to the thickness of the core, however, a relatively long 4 exposure time ( $\sim 40 \mathrm{~s}$ per image) was needed to obtain a reasonable spatial 5 resolution. Hall (2013) used neutron radiography to quantify differential

6 7 8 9 10 11 12 water uptake into an initially air-dry sandstone core that had been subjected to triaxial compression until just after macroscopic failure. Local fluid flow velocities, extracted from image analysis of the neutron radiographs, indicated that water movement was fastest within compacted (low porosity) shear bands. This behavior was attributed to higher capillary forces within the shear bands, possibly due to increased micro-crack density associated with localized damage.

Some of the above observations have been qualitatively interpreted in terms of the dynamics of capillary uptake (Wittman et al., 2010). To date, however, no theoretical framework has been proposed that allows for a quantitative explanation of this phenomenon. The major objectives of this research were to: (i) present a theoretical model for predicting the 1dimensional movement of water into a single air-filled fracture within a porous medium, and (ii) acquire quantitative data on spontaneous imbibition of water in unsaturated fractured Berea sandstone cores using dynamic neutron radiography with which to evaluate the proposed model.

\section{THEORETICAL FRAMEWORK}

The dynamics of water displacing air within a single straight capillary tube are well established (Washburn, 1921). Washburn's model was derived by combining the Young-Laplace equation for the pressure drop across the interface between two static fluids, with the Hagen-Poiseuille equation for the pressure drop in a fluid flowing through a cylindrical tube. Cai et al. 
1 (2010) modified this basic model to predict the dynamics of capillary rise in 2 a single tortuous capillary tube.

$3 \quad$ Here we represent a porous medium as a bundle of non-interacting 4 tortuous capillary tubes, replacing the single tube diameter, $\lambda$, in Cai et al.

5 (2010) with an effective diameter, $\langle\lambda>$, representing the central tendency of 6 the tube diameters within the capillary bundle, i.e. 7

$$
\frac{d L}{d t}=\frac{\langle\lambda\rangle \sigma \cos \theta}{8 \mu \tau^{2}} \frac{1}{L}-\frac{\rho g\langle\lambda\rangle^{2}}{32 \mu \tau^{2}}
$$

9

where $L$ is the height of the wetting front at time $t, \sigma$ is the air-water surface tension, $\theta$ is the water-solid contact angle, $\mu$ is the viscosity of water, $\rho$ is the density of water, $\tau$ is the tortuosity, and $g$ is the gravitational acceleration. Integrating Eq. [1] from $L=0$ at $t=0$ to $L$ at time $t$ yields:

$$
t=-\frac{A}{B^{2}} \ln \left(1-\frac{B}{A} L\right)-\frac{L}{B}
$$

where $A=\langle\lambda\rangle \sigma \cos \theta /\left(8 \mu \tau^{2}\right)$ and $B=\rho g\langle\lambda\rangle^{2} /\left(32 \mu \tau^{2}\right)$. At early times, the effects of gravity are insignificant so that the last term on the right-hand side of Eq. [1] can be neglected. Integrating the resulting expression produces a linear relationship between the wetting front elevation and the square root of time, i.e.

$$
L=C \sqrt{t}
$$

26 (Philip, 1957; Hall, 1989; Reda Taha et al., 2001).

The brittle failure of a loaded porous material can be expected to result in an increase in the effective diameter, $\langle\lambda\rangle$, of the fracture zone as compared 
1 to the unfractured matrix. The tortuosity, $\tau$, of the fracture zone is also likely

2 to be decreased relative to that in the unfractured matrix. The impact of

3 such changes on rates of capillary rise within these two regions can be

4 predicted from Eqs. [2] and [3] as illustrated in Fig. 1. Although increasing

$5<\lambda>$ reduces the late-time equilibrium height of capillary rise in the fracture

6 zone relative to that in the unfractured matrix, water imbibition is markedly

7 increased at early times. This effect is magnified when there is a

8 corresponding reduction in tortuosity of the fracture zone (Fig. 1). Thus,

9 capillary dynamics can explain the more rapid uptake of water in a fracture 10 than in the surrounding matrix during spontaneous imbibition.

11 In addition to capillary uptake within a fracture aperture, rapid wetting

12 can also occur due to surface spreading of water on the side walls of the 13 fracture. This phenomenon was first demonstrated by Tokunaga and Wan 14 (2001), and several recent experimental studies (e.g., Ishino et al., 2007; 15 Vorobyev and Guo, 2010; Liu et al., 2011) have documented the spreading 16 of various liquids over surfaces with different roughness attributes. Hay and 17 Dragila (2008) and Hay et al. (2008) have proposed a theoretical model to explain the physics of fluid spreading on rough surfaces.

We now derive an equation for the rapid early time uptake of water within an air-filled fracture that incorporates both capillarity and surface spreading. Assume $L$ is the height of the wetting front at time $t$ in a smooth-walled fracture. Then the total height of the wetting front, $x$, at time $t$ due to the combination of capillary dynamics and surface spreading effects in a roughwalled fracture is given by (Hay et al., 2008):

$$
x=D \sqrt{t}+L
$$


1 where $D$ is a constant of proportionality that is strongly dependent on the

2 roughness geometry (Hay et al. 2008). Substituting Eq. [3] into Eq. [4] and

3 simplifying the result gives:

4

5

6

7 where $E=(C+D)$ is a lumped sorptivity parameter that combines the

$$
x=D \sqrt{t}+C \sqrt{t}=E \sqrt{t}
$$

effects of both aperture capillarity and the wetting of rough side walls.

Lenormand (1990) studied the pore-scale interfacial geometries resulting from immiscible displacement of one fluid by another in a porous medium. Based on the relative dominance of viscous versus capillary effects the displacement can be categorized as of one of the following types: viscous fingering, capillary fingering, or stable displacement. The displacement of air by water in a homogenous porous medium during spontaneous imbibition results in a uniform wetting front at the macroscopic scale, so that flow can be approximated as a 1-dimensional problem (Kang et al., 2013). The presence of one or more fractures, however, introduces a second flow domain, with water moving more rapidly in the fractures than in the surrounding matrix. Over time the wetting front develops a spatially nonuniform profile with many peaks and depressions (Zhang et al., 2010b). For this reason, macroscopic models for predicting spontaneous imbibition in heterogeneous porous media often include a dispersion parameter to account for capillary dispersion (e.g., Langlo and Espedal, 1994; Zhang and Tchelepi, 1999; Garmeh etal. 2009).

Here we derive a first approximation of the spatial dispersion of the wetting front in a fractured porous medium in terms of the variance of the distances travelled within the matrix and fracture zones, respectively. We consider spontaneous imbibition only at early times (i.e., neglecting the effect of gravity). It is assumed that flow in both the matrix and fracture 
1 zones is 1-dimensional, with no interactions between the two flow domains.

2 From Eq. [3], the distance travelled by the wetting front at time, $t$, in the

3 matrix, $L_{m}$, can be written as:

4

5

$$
L_{m}=C_{m} \sqrt{t}
$$

where $C_{m}$ is the matrix sorptivity. Likewise, from Eq. [5] the distance

travelled by the wetting front at time, $t$, in the fracture zone, $x_{f}$, is given by:

$$
x_{f}=E_{f} \sqrt{t}
$$

12 where $E_{f}$ is the fracture sorptivity. The dispersion coefficient, $\mathbb{D}$, representing

13 the spatial spreading of the wetting front in a heterogeneous porous

14 medium, can be calculated from the variance, $\sigma^{2}$, of the travel distances

15 using the following expression (Fried and Combarnous, 1971; Perfect and

16 Sukop, 2001):

$$
\mathbb{D} \approx \frac{\sigma^{2}}{2 t}
$$

For a dual domain porous medium comprised of an unfractured matrix and a

21 fracture zone, the variance of the travel distances is given by:

$$
\sigma^{2}=\left(\frac{x_{f}-L_{m}}{2}\right)^{2}
$$

25 Substituting Eqs. [6], [7], and [9] into Eq. [8] and simplifying the result 26 produces the following expression for the dispersion of the wetting front 27 during early-time spontaneous imbibition in a fractured porous medium: 28 


$$
\mathbb{D} \approx \frac{1}{8}\left(E_{f}-C_{m}\right)^{2}
$$

3 Equation [10] indicates that the dispersion coefficient is directly proportional

4 to the difference in the sorptivities for the fractured and unfractured zones.

5

6

7

\section{MATERIALS AND METHODS}

\subsection{Rock cores}

Commercially available Berea sandstone cores with different hydraulic properties were investigated. They were purchased from Cleveland Quarries (Vermillion, $\mathrm{OH}$ ). According to Cleveland Quarries the mineralogical composition is of the Berea sandstone is as follows: $93.13 \%$ Silica $\left(\mathrm{SiO}_{2}\right)$, $3.86 \%$ Alumina $\left(\mathrm{Al}_{2} \mathrm{O}_{3}\right), 0.11 \%$ Ferric Oxide $\left(\mathrm{Fe}_{2} \mathrm{O}_{3}\right), 0.54 \%$ Ferrous Oxide (FeO), 0.25\% Magnesium Oxide (MgO), and $0.10 \%$ Calcium Oxide ( $\mathrm{CaO}$ ). The individual cores were $2.54 \mathrm{~cm}$ dia. $\times 5.08 \mathrm{~cm}$ long. They were supplied in three different permeability classes: $\sim 50 \mathrm{mD}, \sim 200 \mathrm{mD}$, and $\sim 500 \mathrm{mD}$. The porosities of the cores, determined from the solid volume measured with a temperature-controlled helium gas pycnometer (HumiPyc Model 1, InstruQuest Inc., Coconut Creek, FL) and the bulk volume measured with a NextEngine Desktop 3D Scanner (NextEngine Inc., Santa Monica, Ca, USA) using the method of Rossi et al. (2008) and Rossi and Graham (2010), were $0.19,0.25$, and 0.24 for the $50 \mathrm{mD}, 200 \mathrm{mD}$, and $500 \mathrm{mD}$ permeability classes, respectively (Table 1 ).

\subsection{Core preparation and fracture induction}

The cores were wrapped in Kapton ${ }^{\circledR}$ tape (DuPont, Wilmington, DE) to provide a no flow barrier along the sides. The wrapped cores were then fractured longitudinally by compression between parallel flat metal plates following the Brazilian method for determining indirect tensile strength ( $\mathrm{Li}$ et 
al., 2013). An example of a typical fracture zone produced by the Brazilian

2 method is shown in Fig. 2.

3

\subsection{Water imbibition setup}

5 Prior to analysis the fractured cores were oven dried at $105^{\circ} \mathrm{C}$ for $24 \mathrm{hr}$. After cooling down they were suspended above a free water table in a high purity aluminum cell that was open to the atmosphere. The aluminum cell was placed in the CG-1D neutron beam line of the Neutron Imaging Prototype Facility at the High Flux Isotope Reactor (HFIR), Oak Ridge National Laboratory (ORNL). The cell was positioned immediately in front of the detector (see below), and was connected to a Mariotte bottle (McCarthy, 1934) located outside of the beam line using Tygon ${ }^{\circledR}$ tubing (Saint-Gobain S.A., La Défense, Courbevoie, France). The water table in the aluminum cell was controlled externally using the Mariotte bottle. The water level was carefully raised over time until it just touched the base of each core. The time of contact was taken as the baseline time, $t=0$, for the imbibition measurements. The height of the water table was then held constant for the duration of each experiment.

\subsection{Dynamic neutron radiography}

Neutrons are ideally suited to the task of visualizing the distribution and movement of water within variably-saturated porous media (Perfect et al., 2014). This is because they are strongly attenuated by the hydrogen atoms in water molecules, but are relative insensitive to the gas phase in pores and minerals (such as silica and iron) which constitute the solid matrix. Digital images of the spatial pattern of neutron attenuation within a sample can be acquired in both 2- and 3-dimensions (2-d and 3-d, respectively). Neutron radiography is the preferred mode of data collection for dynamic processes 
1 such as the rapid uptake of water within fractures since 2-d radiographs can

2 be acquired much more rapidly than 3-d tomograms.

3 Dynamic neutron radiography of the spontaneous imbibition of water

4 displacing air in fractured Berea sandstone cores was performed at CG-1D

5 Neutron Imaging Prototype Facility (HFIR, ORNL). CG-1D is a cold neutron

6 beam line with a curved guide, which eliminates gamma's and epithermal

7 neutrons from the spectrum. The neutron wavelengths vary between 0.8 and

$86 \AA$, with peak intensity at $2.6 \AA$. Apertures with different diameters $(\delta)$ are

9 used at the entrance of the helium-filled flight path (pinhole geometry) to 10 allow for $\ell / \delta$ variation from 400 to 800 , where $\ell$ is the distance between the 11 aperture and the detector. With these $\ell / \delta$ ratios, the beam has a very small 12 angular divergence (a fraction of a degree) and an intensity of $1310^{7}$ neutrons $/ \mathrm{cm}^{2} / \mathrm{s}$.

14 The detector consisted of stacked neutron sensitive microchannel plates 15 (MCP's) placed above a quad Timepix readout with a $28 \times 28 \mathrm{~mm}^{2}$ field of 16 view (FOV) (Tremsin, 2012). The cold neutron detection efficiency is $70 \%$ 17 (Tremsin et al., 2011). The native spatial resolution of the detector is $55 \mu \mathrm{m}$ 18 determined by the pixel size of the Timepix readout, but sub-15 $\mu \mathrm{m}$ spatial 19 resolutions can be achieved through event centroiding (Tremsin et al. 2012). 20 The detector configuration with fast parallel electronics allows readout times 21 of $\sim 290 \mu$ s (Tremsin, 2012; Tremsin et al. 2013) enabling > $1 \mathrm{kHz}$ frame 22 rates. In our experiments, the dry rock cores were imaged at $25 \mu \mathrm{m}$ spatial 23 resolution with the MCP detector in centroiding mode. For the dynamic 24 spontaneous imbibition experiments neutron radiographs were acquired at 25 frequencies of 10-20 frames per second (50-100ms, microsecond) (in order 26 to acquire sufficient number of neutrons per image) with the detector in 27 native spatial resolution mode. 


\subsection{Image analysis and parameter estimation}

The raw neutron radiographs were imported into Image] (Schneider et al., 2012). The high spatial resolution images were normalized with respect to the open beam and despeckled to visualize the rock core and fracture damage zone (Fig. 2). The width of the fracture damage zone was measured at 15 equally-spaced locations along the entire length of the core, and mean values and standard errors were calculated from these measurements. Additionally, images of the top and bottom faces of the fractured cores were acquired with a digital camera, and these images were analyzed in ImageJ. The width of the fracture aperture on each core face was measured at 10 equally-spaced locations. The measurements from both fracture faces were then averaged to provide estimates of the mean fracture aperture width and its standard error.

The wetting images were normalized with respect to the initial image of the time sequence (when the core was completely dry) so that only the imbibing water was resolved (Fig. 3). The resulting normalized images were despeckled and thresholded so that the vertical distance between the water table and the tips of the wetting fronts in the matrix and fracture zone could be quantified over time (Fig. 3).

Matrix and fracture zone sorptivities, $C_{m}$ and $E_{f}$, were estimated from the progression of the wetting front versus square root of time data using linear regression with no intercept. A total of 6 Berea sandstone cores were analyzed: 4 fractured cores (one from each permeability class plus a second core from the $500 \mathrm{mD}$ permeability class to assess between core variability) and 2 unfractured cores (from the 200 and $500 \mathrm{mD}$ permeability classes). Estimates of $C_{m}$ (matrix sorptivity)for the $50 \mathrm{mD}$ and second $500 \mathrm{mD}$ permeability class cores were obtained by analyzing the unfractured matrix on either side of the water-filled fracture zone, well away from the lateral spread of the wetting plume. To assess within core variability, two 
1 measurements of $C_{m}$ were made within the unfractured matrix of each core.

2 Dispersion coefficients for the fractured cores were calculated from the

3 resulting estimates of $C_{m}$ and $E_{f}$ using Eq. [10].

4

\section{RESULTS AND DISCUSSION}

The matrix porosity and fracture characteristics of the Berea sandstone cores are given in Table 1 . The matrix porosity of the $50 \mathrm{mD}$ permeability core was substantially lower than the matrix porosities of the cores in the other permeability classes (Table 1 ).

The Brazilian method created fractures with aperture widths (measured on the top and bottom of the cores) ranging from $120 \mu \mathrm{m}$ to $780 \mu \mathrm{m}$ (Table 1). There was no obvious relationship between the width of the fractures and the matrix porosity or permeability class of the cores. Instead, aperture width and fracture morphology appeared to vary randomly, highlighting the probabilistic nature of the tensile failure process. The $50 \mathrm{mD}$ and second $500 \mathrm{mD}$ permeability class cores produced simple planar fractures that were uniformly narrow (Table 1), whereas the $200 \mathrm{mD}$ and first $500 \mathrm{mD}$ permeability class cores produced complex planar fractures with bifurcations that were much wider and more variable (Table 1 ).

The width of the fracture damage zone (estimated from the neutron radiographs) varied between 2 and 10 times the width of the fracture aperture (Table 1 ). This zone included damage, deformation, and microcracking in addition to the fracture opening. The width of the fracture damage zone generally increased in proportion to the width of the fracture aperture (Table 1). No apparent relationship was found between the width of damage zone and the matrix porosity, or the core permeability class.

Water uptake into all of the fractures was extremely rapid. Figure 3 is a time sequence of neutron radiographs showing water moving into the airfilled fracture damage zone of the $50 \mathrm{mD}$ permeability core. The pore water 
1 pressure was never positive as evidenced by the menisci visible where the 2 sides of the core contact the water table (Fig. 3). At the end of this 3 sequence of images the wetting front in the fracture zone had travelled 4 approximately an order of magnitude further than the wetting front in the 5 matrix (i.e., the dark gray zone at the base of the core).

6 7 9 10 11

The Bond number $(\mathrm{Bo})$ is a dimensionless quantity that can be used to demonstrate that gravity was negligible under the conditions of our experiments. The equation for the Bond number can be written as:

$$
B o=\frac{\left(\rho_{w}-\rho_{n}\right) g k}{\sigma_{w n}}
$$

where $\rho_{w}$ and $\rho_{n}$ are the densities of the wetting and non-wetting fluids, respectively, $k$ is the intrinsic permeability, $\sigma_{w n}$ is the surface tension of the interface between the wetting and non-wetting phases. If $B o<<1$ then gravity can be ignored. For water and air as the wetting and non-wetting fluids, respectively $\left(\rho_{w}=1000 \mathrm{~kg} / \mathrm{m}^{3}, \rho_{n}=0 \mathrm{~kg} / \mathrm{m}^{3}\right.$, and $\sigma_{w n}=0.0728$ $\mathrm{N} / \mathrm{m}$ ) and $g=9.81 \mathrm{~m} / \mathrm{s}^{2}$, the permeability values investigated in this study $\left(k=4.9 \times 10^{-15}\right.$ to $4.9 \times 10^{-14} \mathrm{~m}^{2}$ ) result in calculated $B o$ numbers of between $6.6 \times 10^{-9}$ and $6.6 \times 10^{-8}$ which is much less than unity; so we can safely assume the effect of gravity was negligible under the conditions of our experiments.

Quantitative results from the image analyses of the neutron radiographs are summarized in Figure 4 and Table 1. The spontaneous imbibition of water into both the sandstone matrix and the fracture damage zone exhibited square-root-of-time behavior. This is evidenced by the excellent coefficient of determination $\left(R^{2}\right)$ values (i.e. $>0.9$ ) for Eqs. [6] and [7] fitted to the experimental observations regardless of the location of the uptake (Fig. 4). It can be seen that the first point of some of the data sets (e.g., the fracture zone in Fig. $4 a$ and the matrix in Fig. 4c) tends to fall 
1 below the best fit line. These outliers indicate deviations from square-root-

2 of-time behavior at very early times. Possible causes of these deviations

3 are: (1) the base of the core may not have be perfectly aligned with the 4 water surface resulting in partial contact at very early times, and (2) the

5 first image was integrated over a relatively long period of time (5 to $10 \mathrm{~ms}$ ) 6 relative to the rate of wetting at very early times.

7 Between core variability of the matrix and fracture zone water uptake 8 measurements can be assessed by comparing the results for the two 500 $9 \mathrm{mD}$ permeability class cores in Figs. 4(c) and 4(d). In general, both sets of 10 measurements were reasonably comparable; coefficients of variation (CV) 11 calculated from the slopes of the regression equations for the two cores 12 were $6 \%$ and $12 \%$ for the matrix and fracture zone data, respectively. In 13 some respects, one might have expected greater variability between the 14 fracture imbibition measurements for these two cores due to the different 15 aperture and damage zone widths (Table 1 ).

The replicate measurements of spontaneous imbibition of water into the unfractured matrix of each core permit an assessment of the within core variability for this measurement. The results in Fig. 4 indicate generally good reproducibility; the $50 \mathrm{mD}$ permeability class core (Fig. 4a) exhibited the greatest variability with a CV of $17 \%$, while the CV's for the other three cores were all $<2.5 \%$. Since there was only one fracture damage zone per core it was not possible to assess within-core variability for this parameter.

The matrix sorptivities ranged from 2.90 to $4.55 \mathrm{~mm} \mathrm{~s}^{-0.5}$ (Table 1), and increased linearly as the permeability class increased (Fig. 5a). The matrix sorptivity coefficient changed by a factor of $\sim 2$ as the permeability changed by a factor of $\sim 4$ which is consistent with the traditional assumed square dependence of the permeability on the mean pore size (Table 1).

The average value for the $50 \mathrm{mD}$ permeability class core was $\sim 2.5$ times higher than the average matrix sorptivity for Berea sandstone determined 
1 by Kang et al. (2013) using the same method. The cores analyzed by Kang 2 et al. (2013) were supplied by a different company and had a slightly lower 3 average permeability of $\sim 42 \mathrm{mD}$. Moreover, due to differences in the 4 detector systems employed by the two studies, water uptake data were 5 collected over two completely different time frames. In the present study, 6 measurements were made over the range $0.1 \sim 2.0 \mathrm{~s}$, whereas the Kang et 7 al. (2013) data were obtained over the range 5 50 s with a charge-coupled 8 device (CCD) detector at the same beam line. Thus, the lower matrix 9 sorptivities reported in Kang et al. (2013), as compared to those in Table 1 10 can be attributed to a combination of lower core permeability and the 11 increased effect of gravity at later times as described by Eq. [2]. 12 The fracture damage zone sorptivities were between 4 and 7 times 13 greater than the corresponding matrix sorptivities (Table 1 ). The sorptivities 14 of the fracture damage zones ranged from 17.87 to $27.12 \mathrm{~mm} \mathrm{~s}^{-0.5}$ (Table 15 1), and increased linearly with increasing fracture aperture width (Fig. 5b). 16 The fracture sorptivity coefficients for the $50 \mathrm{md}$ and $500 \mathrm{md}$ (replicate \#2) 17 permeability cores samples were similar, which is consistent with the similar 18 fracture aperture widths in these two samples (Table 1 ).

19 The sorptivity results indicate that fractures can significantly increase 20 spontaneous imbibition in unsaturated sedimentary rock by capillary action 21 and surface spreading on rough fracture faces. Our results are consistent 22 with the studies by Şahmaran and Li (2009) and Zhang et al. (2010a,b), 23 which showed enhanced sorption in fractured cementitious materials. The 24 rates of water uptake observed in the present study were surprisingly rapid. 25 The fracture sorptivities in Table 1 indicate that wetting fronts can travel 26 between 2 and $3 \mathrm{~cm}$ in 1 second in unsaturated damage zones. 27 We ran a simple qualitative experiment to isolate the phenomenon of 28 water spreading over a rough fracture surface, Eq. [4] from the dynamics of 29 capillary rise within a narrow fracture aperture, Eq. [3]. An oven-dry 500 
$1 \mathrm{mD}$ permeability class Berea sandstone core was fractured as described

2 previously. The two halves of the fractured rock core were carefully

3 separated and one half was rotated $180^{\circ}$ relative to the other half. The two

4 halves were then placed side-by-side, at the same time, in a shallow pan of

5 water so that water uptake could be monitored simultaneously on the

6 interior (fracture face) and exterior surfaces of the core.

7 Figure 6 shows the results of spontaneous imbibition of water into the 8 two halves of the fractured core after approximately $\sim 1-2 \mathrm{~s}$ of wetting. It 9 can be clearly seen that the wetting front has moved approximately twice 10 as far on the rough fracture surface as compared to the wetting front on the 11 smooth exterior of the core. This movement occurred in the absence of the 12 second fracture face, indicating that water spread over the rough surface of 13 the exposed fracture face, similar to the observations of Tokunaga and Wan 14 (2001) and Vorobyev and Guo (2010). The wetting front on the other half 15 of the core, with its smooth exterior exposed, can be attributed to capillary 16 rise within the unfractured matrix. It is interesting to note that the wetting 17 front on the exterior of the core was curvilinear. This was likely due to 18 surface spreading on the (unseen) fracture face on the other side of this 19 half of the core which has induced water to move more rapidly along the 20 edges. Overall, this simple experiment demonstrates that surface spreading 21 contributes significantly to the rapid imbibition of water in fractured 22 sedimentary rocks.

23 The dispersion coefficients, calculated from the matrix and fracture 24 sorptivities, ranged from 23.7 to $66.7 \mathrm{~mm}^{2} \mathrm{~s}^{-1}$ (Table 1 ). Figures 5(c) and $255(d)$ indicate that dispersion increased linearly with increasing width of both 26 the fracture aperture and its associated damage zone. The strongest 27 relationship was with the fracture aperture width. The larger the dispersion 28 parameter, the greater the spreading of the wetting front within the core. 29 


\section{CONCLUDING REMARKS}

Neutron radiography was able to capture the early-time dynamics of spontaneous imbibition of water in fractured, unsaturated sedimentary rock cores. Rates of water uptake into the fracture damage zones were surprisingly rapid, with wetting fronts travelling between 2 and $3 \mathrm{~cm}$ per second. A theoretical model was derived to explain these results. The rapid water uptake was attributed to a combination of early time capillary rise within the fracture aperture and spreading over the rough surfaces of the opposing fracture faces. The theory also permits estimation of sorptivity values for the matrix and fracture zones, as well as a dispersion parameter, which quantifies the extent of spreading of the wetting front. The sorptivity of the fracture zone and the dispersion coefficient both increased linearly with increasing fracture aperture width.

The current theoretical framework represents only a 1-dimensional approximation of spontaneous imbibition within a fractured porous medium. To fully describe this phenomenon the derivation of a 2-dimensional model, that permits interaction between the fracture and matrix, will be needed. The development of such a model represents an exciting opportunity for future research.

The present results for fractured Berea sandstone are consistent with observations of rapid water uptake in fractured cementitious materials. Additional research is needed to document this phenomenon in other natural and engineered porous media. The phenomenon is likely to be particularly important in the case of porous materials such as bricks and building stone used in engineering applications. Given the current high level of interest in unconventional gas from black shale, similar experiments performed on fractured shale cores may also be valuable. The spontaneous imbibition of water-based fracking fluids into damage zones created by 
1 hydraulic fracturing operations could potentially result in significant losses

2 of water ("leakoff") with concomitant blockages of the pathways available

3 for efficient gas extraction.

4

\section{ACKNOWLEDGEMENTS}

E. Perfect acknowledges support from David E. Jackson of BDY

Environmental LLC, Nashville, TN through a Faculty Achievement Award. Research conducted at ORNL's High Flux Isotope Reactor was sponsored by the Scientific User Facilities Division, Office of Basic Energy Sciences, U.S. Department of Energy.

\section{REFERENCES}

Cai, J.C., B.M. Yu, M.F. Mei, and L. Luo. 2010. Capillary rise in a single tortuous capillary. Chin. Phys. Lett. 27: 054701.

Chen, Q., and W. Kinzelbach. 2002. An NMR study of single- and two-phase flow in fault gouge filled fractures. J. Hydrol. 259: 236-245.

Fried, J.J., and M.A. Combarnous. 1971. Dispersion in porous media. Adv. Hydroscience 7: 169-282.

Garmeh, G., R.T. Johns, and L.W. Lake. 2009. Pore-scale simulation of dispersion in porous media. SPE J. 14: 559-567.

Hall, C. Water sorptivity of mortars and concrete: a review. Mag. Concrete Res. 41: 51-61.

Hall, S.A. 2013. Characterization of fluid flow in a shear band in porous rock using neutron radiography. Geophys. Res. Lett. 40: 1-6.

Hay, K.M., and M.I. Dragila. 2008. Physics of fluid spreading on rough surfaces. Intl. J. Num. Anal. Model. 5: 85-92.

Hay, K.M., M.I. Dragila, and J. Liburdy. 2008. Theoretical model for the wetting of a rough surface. J. Colloid Interface Sci. 325: 472-477 
1 Ishino, C., M. Reyssat, E. reyssat, K. okumura, and D. Quéré. 2007. Wicking within forests of micropillars. Europhys. Lett. 79: 56005.

Kanematsu, M., I. Maruyama, T. Noguchi, H. Iikura, and N. Tsuchiya. 2009. Quantification of water penetration into concrete through cracks by neutron radiography. Nuclear Instrum. Meth. Physics Res. A605: 154158.

Kang, M., E. Perfect, C.L. Cheng, H.Z. Bilheux, M. Gragg, D.M. Wright, J.M. Lamanna, J. Horita, and J.M. Warren. 2013. Diffusivity and sorptivity of Berea sandstone determined using neutron radiography. Vadose Zone J. 12: doi:10.2136/vzj2012.0135.

Karpyn, Z.T., P.M. Halleck, A.S. Grader. 2009. An experimental study of spontaneous imbibition in fractured sandstone with contrasting sedimentary layers. J. Petrol. Sci. Eng. 67: 48-56.

Langlo, P., and M.S. Espedal. 1994. Macrodispersion for two-phase, immiscible flow in porous media. Adv. Water Resour. 17: 297-316.

Lenormand, R. 1990. Liquids in porous media. J. Phys. Condens. Matter 2: SA79-SA88.

Li, D., L. Ngai, and Y. Wong. 2013. The Brazilian disc test for rock mechanics applications: Review and new insights. Rock Mech. Rock Eng. 46: 269-287.

Liu, W., Y.Li, Y. Cai, and D.P. Sekulic. 2011. Capillary rise of liquids over microstructured solid surface. Langmuir 27: 14260-14266.

Lunati, I., P. Vontobel, W. Kinzelbach, and E. Lehmann. 2003. Laboratory visualization of two-phase flow in a natural fracture by neutron tomography. Conference on Groundwater in Fractured Rocks, Prague, Czech Republic, September 15-19, 2003, 2 pp.

McCarthy, E. L. 1934. Mariotte's bottle. Science 80: 100.

Perfect, E., and M.C. Sukop. 2001. Models relating solute dispersion to pore space geometry in saturated media: A review. In: H.M. Selim and 
D.L. Sparks (eds.), Physical and Chemical Processes of Water and Solute Transport / Retention in Soil, Special Publ. 56, Soil Sci. Soc. Am., Madison WI, p.77-146.

Perfect, E., C.-L. Cheng, M. Kang, H.Z. Bilheux, J.M. Lamanna, M.J. Gragg, and D.M. Wright. 2014. Neutron imaging of hydrogen-rich fluids in geomaterials and engineered porous media: A review. Earth Sci. Rev. 129:120-135.

Philip, J.R. 1957. The theory of infiltration: 4. Sorptivity and algebraic infiltration equations. Soil Sci. 84: 257-264.

Polsky, Y., L.M. Anovitz, P. Bingham, and J. Carmichael. 2013. Application of neutron imaging to investigate flow through fractures for EGS. Proc. 38th Workshop on Geothermal Reservoir Engineering, Stanford Univ., Stanford, CA, Feb, 11-13, 2013, https://pangea.stanford.edu/ ERE/pdf/IGAstandard/SGW/2013/Polsky1.pdf, Accessed 6/16/14.

Rangel-German, E.R., and A.R. Kovscek. 2002. Experimental and analytical study of multidimensional imbibition in fractured porous media. $\mathrm{J}$. Petrol. Sci. Eng. 36: 45-60.

Reda Taha, M.M., A.S. El-Dieb, and N.G. Shrive. 2001. Sorptivity: a reliable measurement for surface absorption of masonry brick units. Mater. Struct. 34: 438-445.

Rossi, A.M., D.R. Hirmas, R.C. Graham, P.D. Sternberg. 2008. Bulk density determination by automated three-dimensional laser scanning. Soil Sci. Soc. Am. J. 72: 1591-1593.

Rossi, A. M., and R. C. Graham. 2010. Weathering and porosity formation in subsoil granitic clasts, Bishop Creek Moraines, California. Soil Sci. Soc. Am. J. 74: 172-185.

Şahmaran, M., and V.C. Li. 2009. Influence of microcracking on water absorption and sorptivity of ECC. Mater. Struct. 42: 593-603. 
Schneider, C.A., W.S. Rasband, and K.W. Eliceiri. 2012. NIH Image to ImageJ: 25 years of image analysis. Nature Methods 9: 671-675.

Snoeck, D., S. Steuperaert, K. Van Tittelboom, P. Dubruel, and N. De Belie. 2012. Visualization of water penetration in cementitious materials with superabsorbent polymers by means of neutron radiography. Cement Concrete Res. 42: 1113-1121.

Tokunaga, T.K., and J. Wan. 2001. Surface-zone flow along unsaturated rock fractures. Water Resour. Res. 37: 287-296.

Tremsin, A.S., J.B. McPhate, J.V. Vallerga, O.H.W. Siegmund, W.B. Feller, and E. Lehmann. 2011. Improved efficiency of high resolution thermal and cold neutron imaging. Nucl. Instr. Meth. A 628: 415-418.

Tremsin, A.S., 2012. High resolution microchannel plates neutron counting detectors and their applications in neutron radiography, diffraction and resonance absorption imaging. Neutron News 23: 35-38.

Tremsin, A.S., J.B. McPhate, J.V. Vallerga, O.H.W. Siegmund, W.B. Feller, E. Lehmann, A .Kaestner, P. Boillat, T. Panzner, and U. Filges. 2012. Neutron radiography with sub-15 $\mu \mathrm{m}$ resolution through event centroiding. Nucl. Instr. Meth. A 688: 32-40.

Tremsin, A.S., J.V. Vallerga, J.B. McPhate, O.H.W. Siegmund, R. Raffanti. 2013. High resolution photon counting with MCP-Timepix quad parallel readout operating at $>1 \mathrm{kHz}$ frame rates. IEEE Trans. Nucl. Sci. 60: 578-585.

Van Tittelboom, K., D. Snoeck, P. Vontobel, F.H. Wittmann, and N. De Belie. 2013. Use of neutron radiography and tomography to visualize the autonomous crack sealing efficiency in cementitious materials. Mat. Struct. 46:105-121.

Vorobyev, A.Y., and C. Guo. 2010. Water sprints uphill on glass. J. appl. Phys. 108: 123512.

Washburn, E.W. 1921. Dynamics of capillary flow. Phys. Rev. 17: 273-283 
1 Wittmann, F.H., P. Zhang, T. Zhao, E. Lehmann, and P. Vontobel. 2010.

2 Neutron radiography, a powerful method for investigating water

3 penetration into concrete. p. 61-70 In: C. Miao, G. Ye, and H. Chen

4 (eds.), Advances in Civil Engineering Materials, Proceedings, 50 years

5 Teaching and Research of Professor Sun Wei, Pro071, RILEM

6 Publications SARL, Bagneux, France.

7 Zhang, D. and H. Tchelepi. 1999. Stochastic analysis of immiscible two-

8 phase flow in heterogeneous media. SPE J. 4: 380-388

9 Zhang, P., F. Wittmann, T. Zhoa, and E. Lehmann. 2010a. Neutron imaging 10 of water penetration into cracked steel reinforced concrete. Physica $B$

17 Zhang, P., F.H. Wittmann, T. Zhao, E.H. Lehmann, and P. Vontobel, 2011. 405: 1866-1871.

Zhang, P., F. Wittmann, T. Zhoa, E. Lehmann, L. Tian, and P. Vontobel. 2010b. Observation and quantification of water penetration into strain hardening cement-based composites (SHCC) with multiple cracks by means of neutron radiography. Nucl. Instr. Meth. Phys. Res. A 620: 414-420. Neutron radiography, a powerful method to determine timedependent moisture distributions in concrete. Nuclear Engineering and Design. 241: 4758-4766. 
Table 1. Mean values and associated standard errors (in parentheses) of measured hydraulic parameters for the fractured Berea sandstone cores.

\begin{tabular}{ccccccc}
\hline $\begin{array}{c}\text { Permeability } \\
\text { class }(\mathrm{mD})\end{array}$ & $\begin{array}{c}\text { Matrix } \\
\text { porosity } \\
\left(\mathrm{m}^{3} \mathrm{~m}^{-3}\right)\end{array}$ & $\begin{array}{c}\text { Matrix } \\
\text { sorptivity } \\
\left(\mathrm{mm} \mathrm{s}^{-0.5}\right)\end{array}$ & $\begin{array}{c}\text { Fracture } \\
\text { aperture } \\
\text { width }(\mu \mathrm{m})\end{array}$ & $\begin{array}{c}\text { Fracture } \\
\text { damage zone } \\
\text { width }(\mu \mathrm{m})\end{array}$ & $\begin{array}{c}\text { Fracture zone } \\
\text { sorptivity } \\
\left(\mathrm{mm} \mathrm{s}^{-0.5}\right)\end{array}$ & $\begin{array}{c}\text { Dispersion } \\
\text { coefficient } \\
\left(\mathrm{mm}^{2} \mathrm{~s}^{-1}\right)\end{array}$ \\
\hline 50 & $\begin{array}{c}0.19 \\
(0.01)\end{array}$ & $\begin{array}{c}2.90 \\
(0.21)\end{array}$ & $\begin{array}{c}140 \\
(20)\end{array}$ & $\begin{array}{c}620 \\
(80)\end{array}$ & $\begin{array}{c}17.87 \\
(0.78)\end{array}$ & $\begin{array}{c}28.01 \\
(3.71)\end{array}$ \\
& 0.25 & 4.05 & 780 & 1,610 & 27.12 & 66.53 \\
200 & $(0.01)$ & $(0.19)$ & $(120)$ & $(100)$ & $(0.96)$ & $(6.63)$ \\
500 & 0.24 & 3.77 & 350 & 1,310 & 19.86 & 32.36 \\
(replicate 1) & $(<0.01)$ & $(0.24)$ & $(50)$ & $(80)$ & $(0.85)$ & $(4.38)$ \\
500 & 0.24 & 4.55 & 120 & 1,170 & 18.32 & 23.70 \\
(replicate 2) & $(<0.01)$ & $(0.25)$ & $(10)$ & $(130)$ & $(0.54)$ & $(2.72)$ \\
\hline
\end{tabular}

${ }^{a}$ Average of the two replicate measurements reported in Fig. 4 
FIGURE CAPTIONS

Figure 1. Wetting front elevation versus time predicted using Eqs. [2] and [3] for a two-region porous medium with $\langle\lambda\rangle=50 \mu \mathrm{m}$ and $\tau=1.8$ in the unfractured matrix and $\langle\lambda\rangle=150 \mu \mathrm{m}$ and $\tau=1.2$ in the fracture zone. All of the other parameters in Eqs. [2] and [3] were the same for the two regions, i.e. $\sigma=0.0728 \mathrm{~N} / \mathrm{m}, \mu=0.001 \mathrm{Ns} / \mathrm{m}^{2}, \cos (\theta)=$ $1, \rho=1000 \mathrm{~kg} / \mathrm{m}^{3}$, and $g=9.81 \mathrm{~m} / \mathrm{s}^{2}$.

Figure 2. Example of a longitudinal air-filled fracture zone (dark gray) in an oven dry $50 \mathrm{mD}$ permeability class Berea sandstone core (light gray) imaged using neutron radiography at the HFIR's CG-1D beam line, ORNL (see Kang et al., 2013 for technical details). For scale, the core is $2.54 \mathrm{~cm}$ dia. $\times 5.08 \mathrm{~cm}$ long. The aluminum container and basal reservoir can also be seen in outline. The core was wrapped in Kapton tape to provide a no flow barrier along the sides. The top of the core was wrapped more thickly than the bottom resulting in the contrasting lighter and darker sections. The $28 \times 28 \mathrm{~mm}^{2} \mathrm{FOV}$ for the fracture imbibition images in Fig. 3 is shown as a white box.

Figure 3. Time sequence of neutron radiographs running from left to right and top to bottom showing the rapid uptake of water into a longitudinal, air-filled fracture zone in Berea sandstone ( 50 mD). The images have been normalized with respect to the initial dry image, so that only water (black) is visible. The rock core, similar to the one in Figure 2, was positioned above a water table which contacted its base immediately after the first image. The height of the water table was then held constant. The annotations on the $1.4 \mathrm{sec}$ image indicate where the distances traveled by the wetting front in 
the matrix, $L_{m}$, (2 replicate locations) and fracture zone, $x_{f}$, were measured. For scale the width of each image is $26 \mathrm{~mm}$.

3

Figure 5. Linear regression relationships between the sorptivity and dispersion estimates and core permeability class and fracture parameters: (a) matrix sorptivity versus permeability class, (b) fracture zone sorptivity versus fracture aperture width, (c) dispersion coefficient versus fracture aperture width, and (d) dispersion coefficient versus fracture zone width. The horizontal and vertical bars around the individual data points represent plus and minus standard errors.

Figure 6. Spontaneous imbibition of water into a fractured, oven dry 500 $\mathrm{mD}$ permeability class Berea sandstone core showing the differential extent of wetting on the rough interior fracture surface as compared to the smooth exterior surface. 


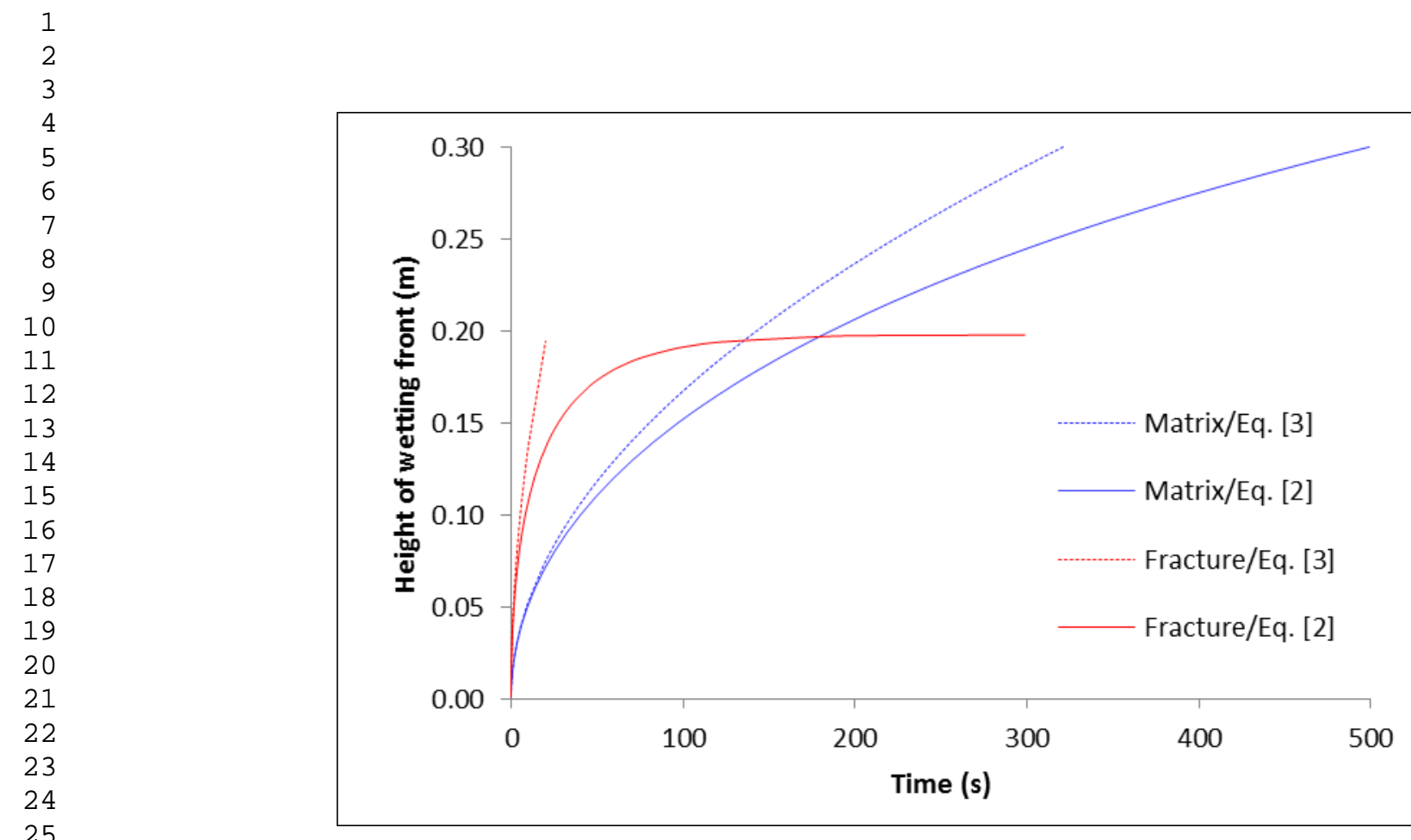

Fig. 1

29

30

31

32

33

34

35

36

37

38

39

40

41

42

43

44

45

46

47

48

49

50

51

52

53

54

55

56

57

58

59

60

61

62

63

64

65 


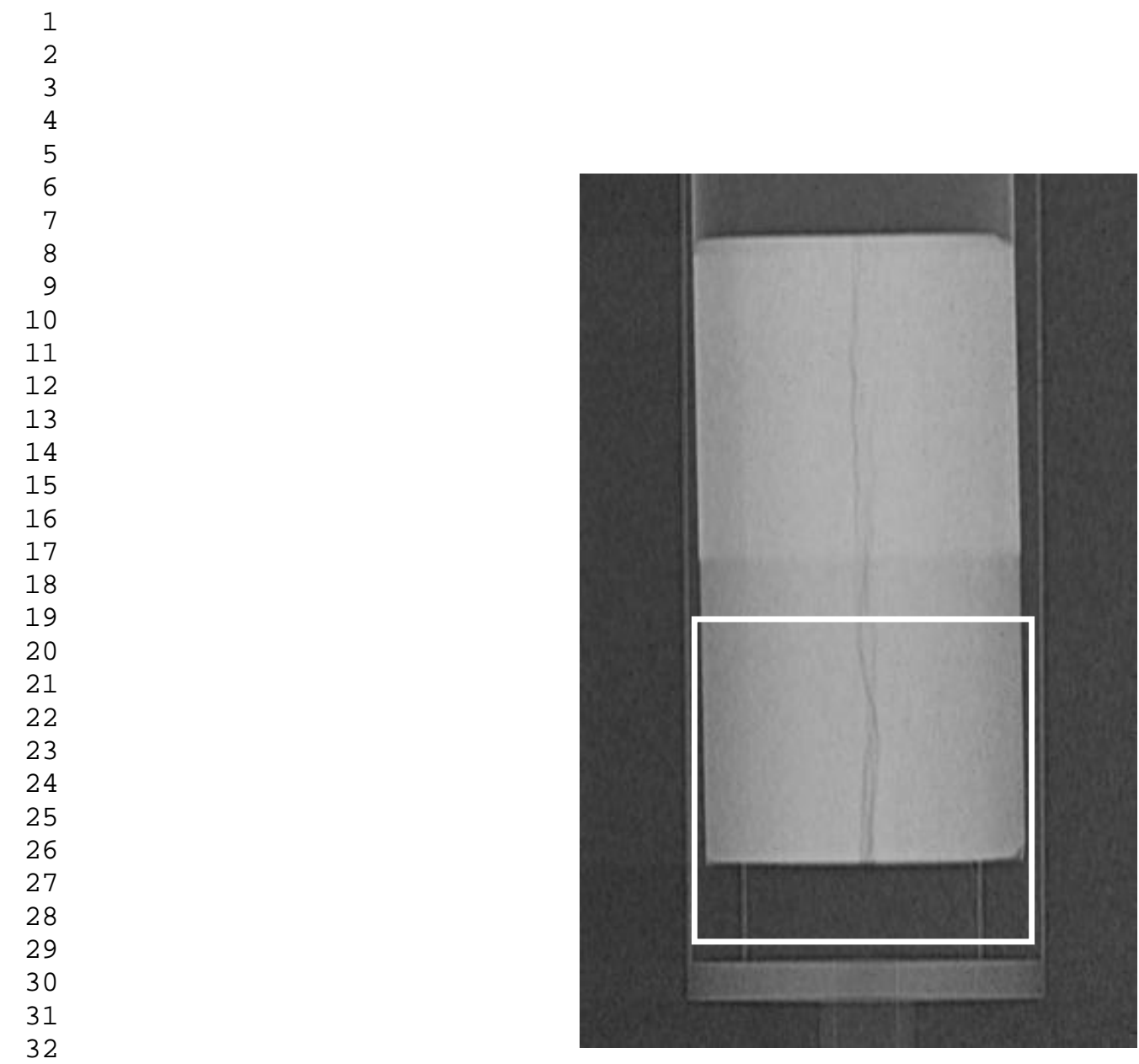

Fig. 2 


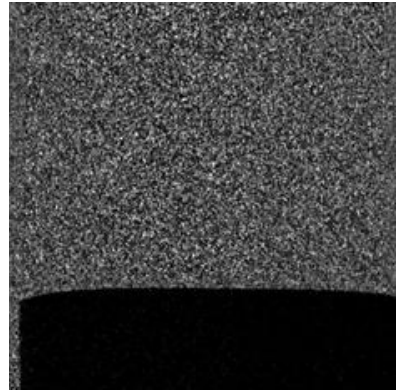

$0 \mathrm{sec}$

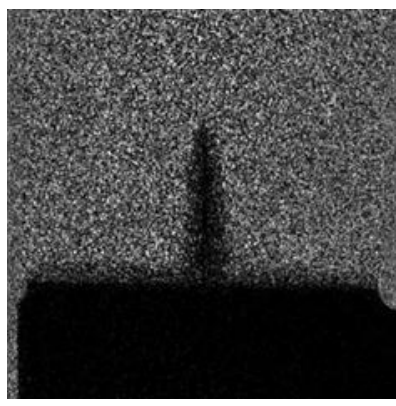

$0.5 \mathrm{sec}$

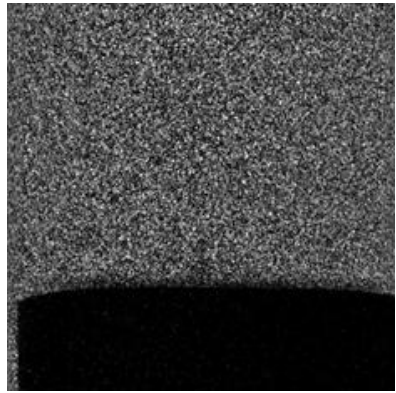

$0.1 \mathrm{sec}$

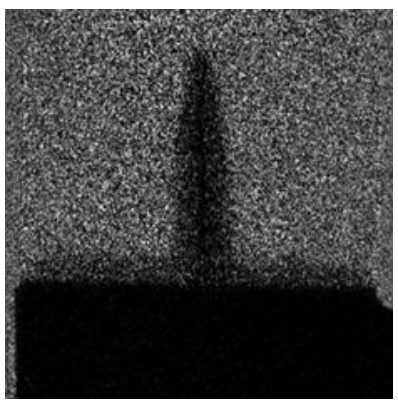

$1.0 \mathrm{sec}$

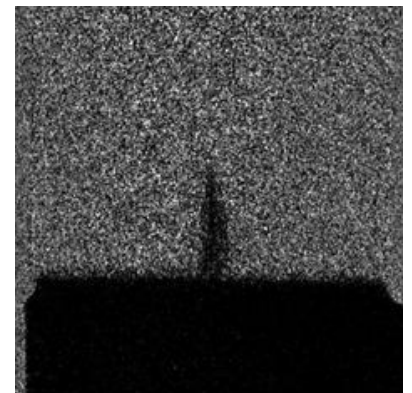

$0.2 \mathrm{sec}$

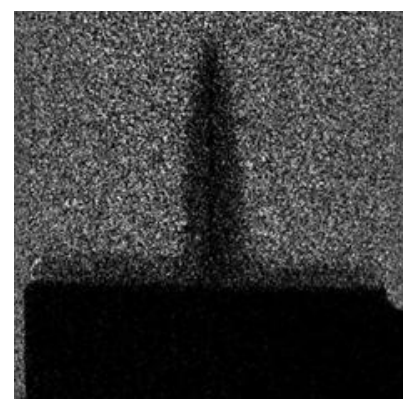

$1.2 \mathrm{sec}$

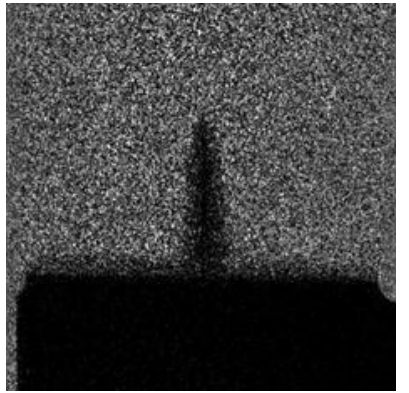

$0.3 \mathrm{sec}$

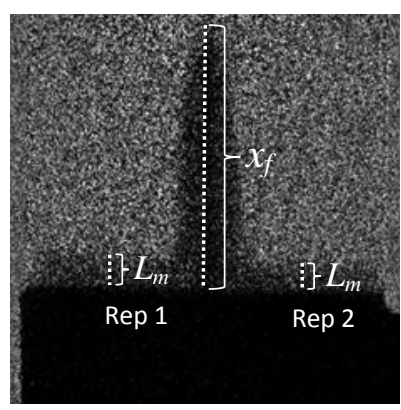

$1.4 \mathrm{sec}$

Fig. 3 


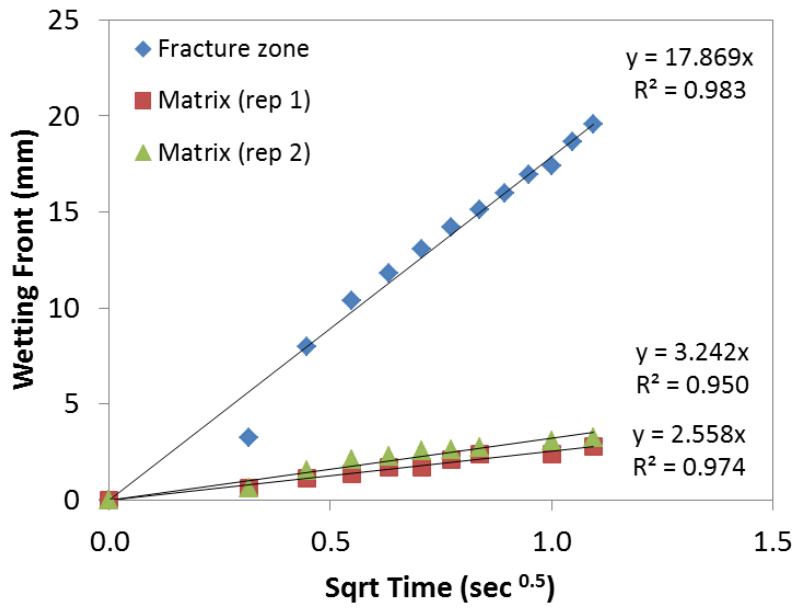

(a)

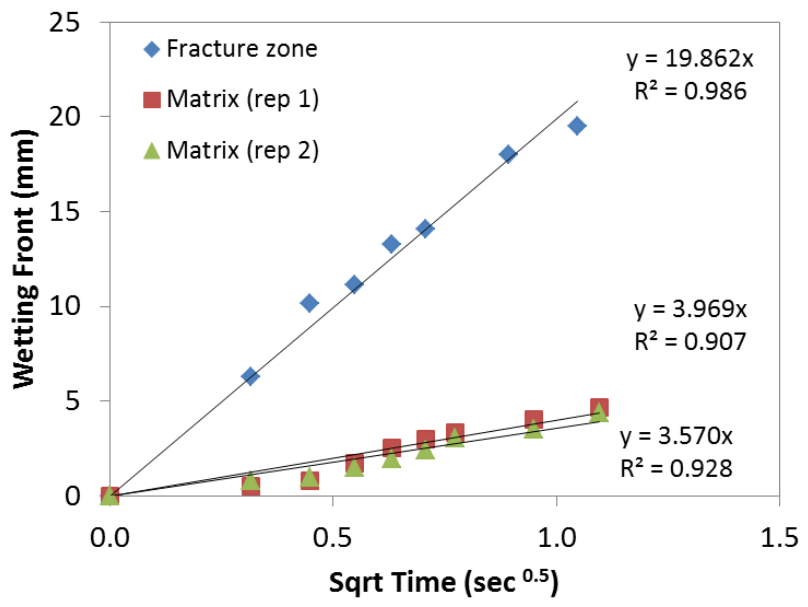

(c)

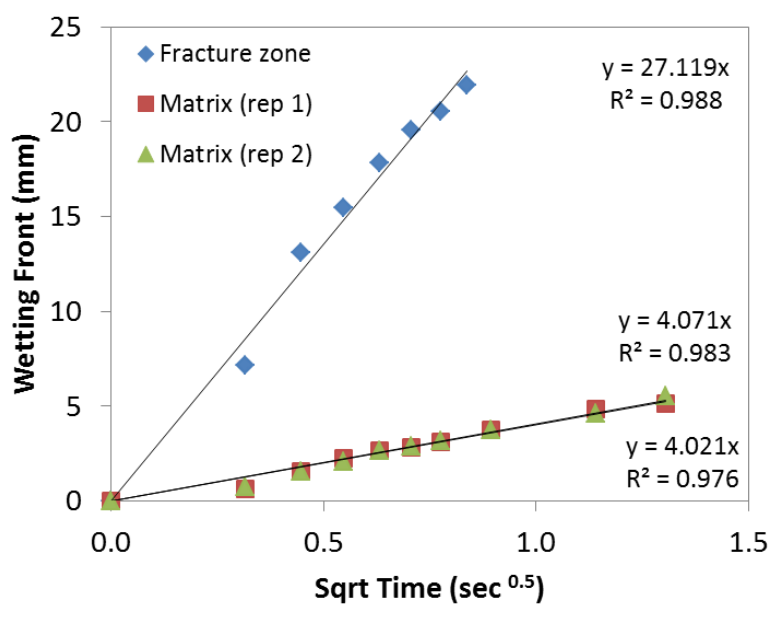

(b)

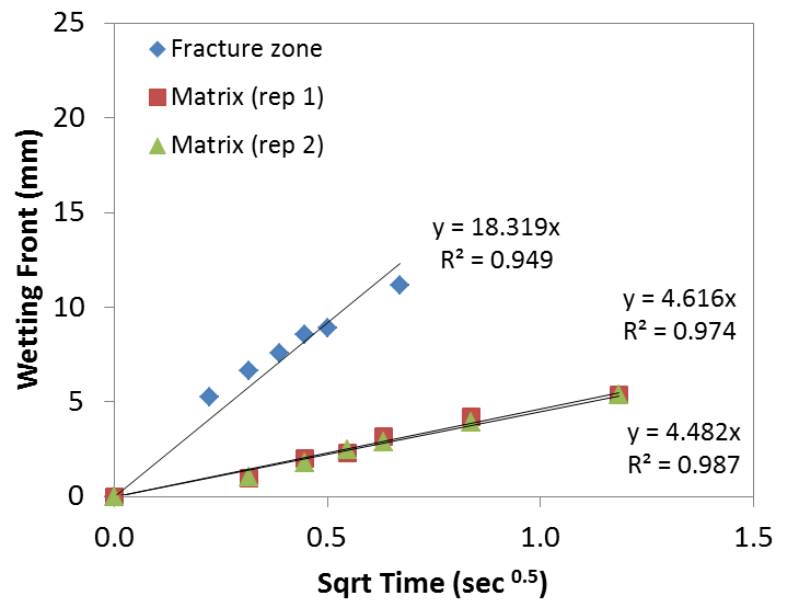

(d)

Fig. 4 


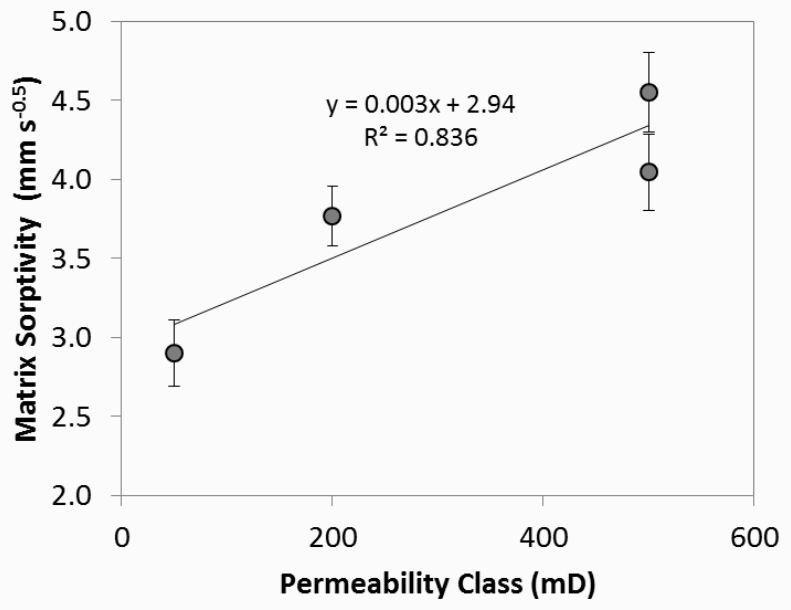

(a)

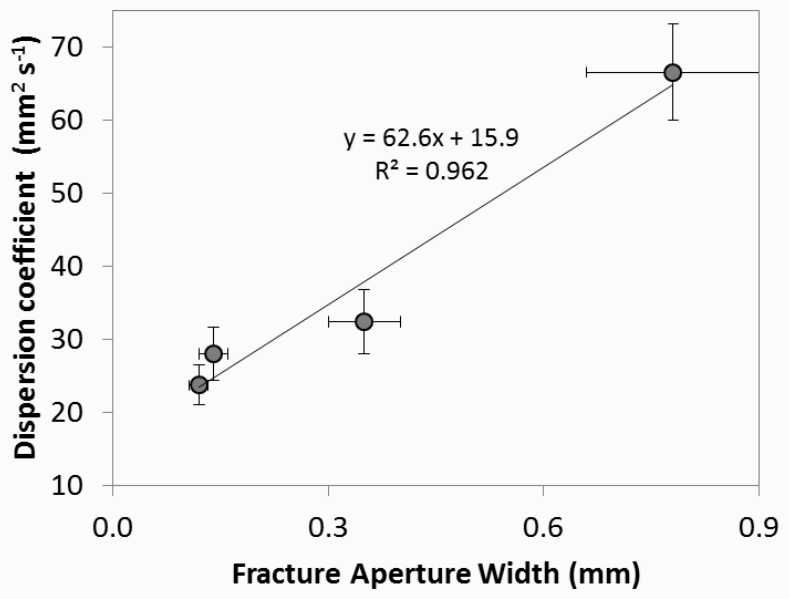

(c)

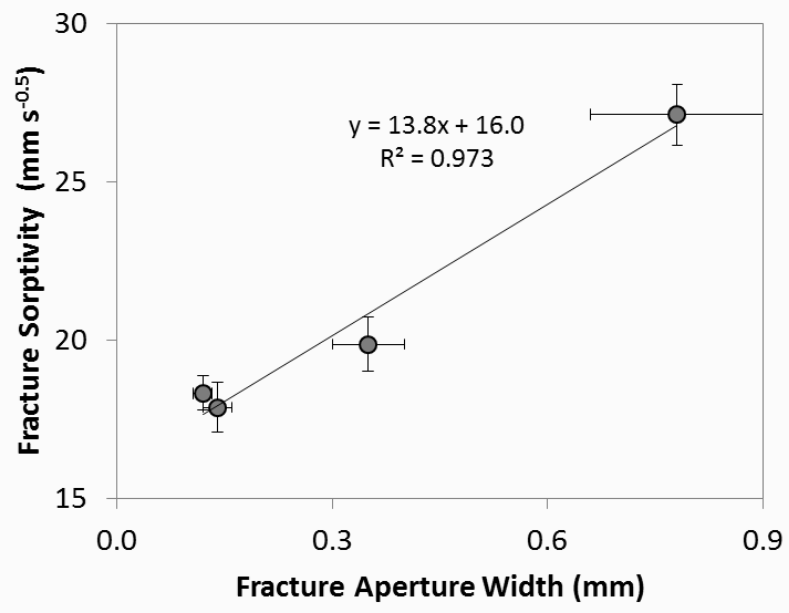

(b)

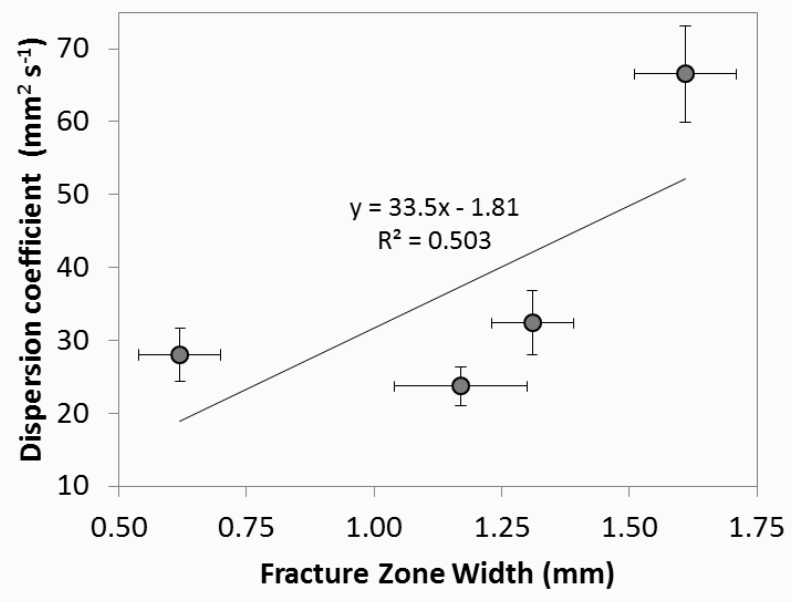

(d)

Fig. 5 


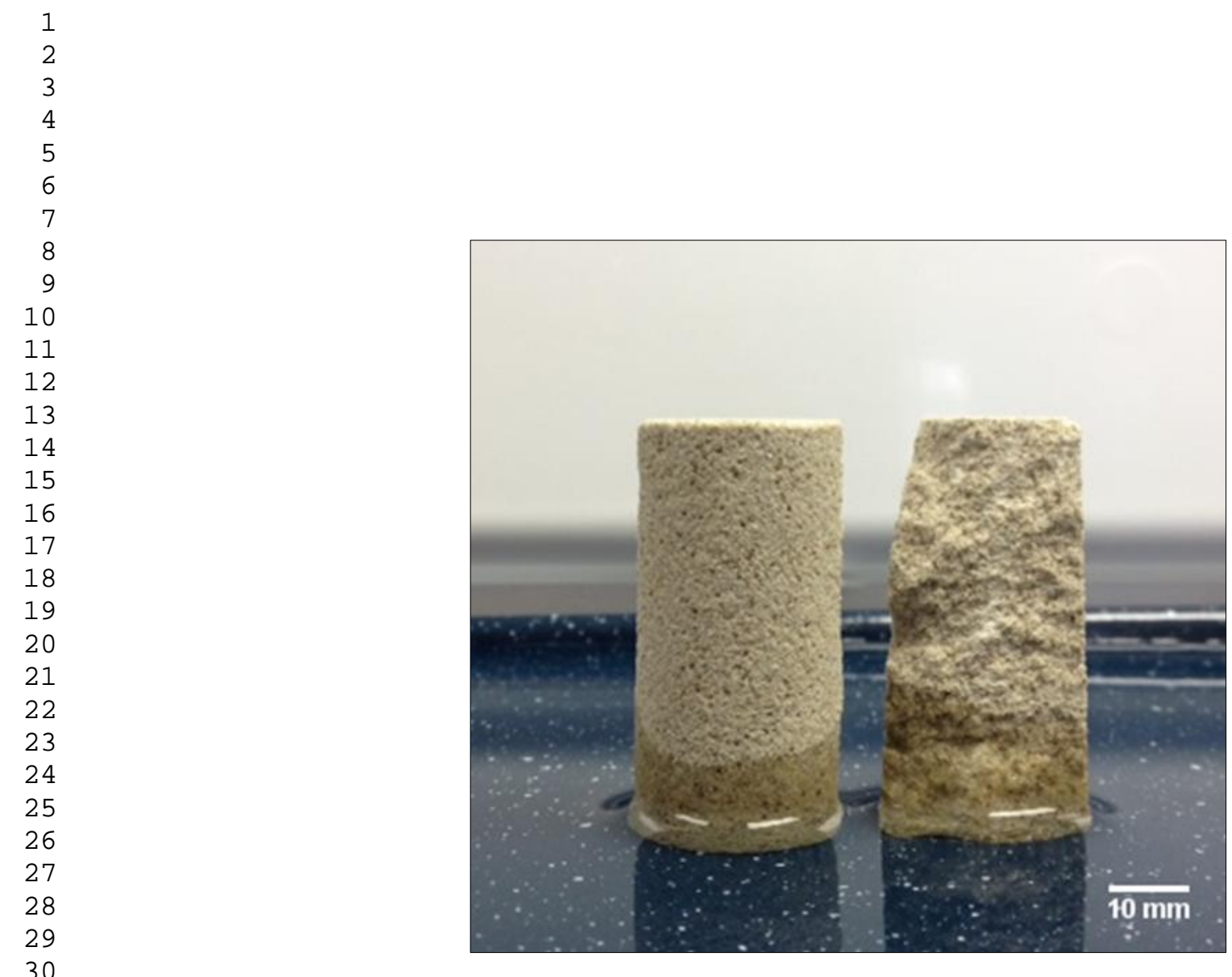

33

34

35

36

37

38

39

40

41

42

43

44

45

46

47

48

49

50

51

52

53

54

55

56

57

58

59

60

61

62

63

Fig. 6 